$\xi=-1$

\title{
A survey: issues and challenges in wireless sensor network
}

\author{
Tarun Bala *, Varsha Bhatia, Sunita Kumawat, Vivek Jaglan \\ Amity University Haryana, India \\ *Corresponding author E-mail: tarun.arora1278@yahoo.in
}

\begin{abstract}
Wireless Sensor Network (WSN) is powerful and economical key to monitoring and tracking problems. WSN is a perfect unification of sensing, computation and communications tasks. WSN nodes are deployed in both hostile and harsh environment. They lend valuable support for continuous, remote monitoring, and for healthcare applications. WSN nodes can be deployed easily in existing structures without any added infrastructure. In this work, an outline of wireless sensor networks, applications, challenges \& issues involved in implementing them is presented.
\end{abstract}

Keywords: Wireless Sensor Networks (WSN), WSN classification, Issues, Challenges.

\section{Introduction}

In recent years, the advancement of WSN has increased at a rapid pace. A typical WSN comprises of large number of small sensor nodes, they monitor or sense physical attribute such as temperature or pressure, process data and communicate this data to base station or sink. Generally the network is static and batterypowered. WSN nodes are capable of organizing themselves into cooperative network. These nodes are deployed in large numbers to monitor or track events, normally in regions which lack connectivity \& wired internet infrastructure. Once deployed, these nodes can work without human interference.

WSN can be deployed in structured or unstructured or random way. Type of deployment depends on WSN application and deployment terrain. In harsh environment and hard to reach places random deployment is the only possibility, but care must be taken to avoid uncovered regions. In structured deployment nodes are placed at specific location, hence fewer nodes are required and their maintenance, management and redeployment of nodes become easy [2].

WSN is resource constraint environment as compared to traditional wireless network, in terms bandwidth, power, storage \& processing capability [3]. WSN design constraint depends on the application and environment to be monitored. For any application, making WSN implementation a reality, three basic attributes are required. These are low cost, lesser power and real time support. WSN nodes are deployed in large number, to make it financially feasible the cost of sensors and their deployment must be low [2]. Redeployment or recharging sensor nodes in large scale network is troublesome and costly; hence focus should be on designing low power sensor nodes and minimizing the power consumption of sensor nodes.WSN networks are used for varied kind of applications, such as constant monitoring applications, event detection applications and time critical applications. Data reporting for such application is done periodically, based on happening of any Event, or only in time critical situations respectively.

Event detection and time critical applications require real time support, where delay and latency are intolerable.
Application of Wireless Sensor Nodes: WSN can be applied to diverse domains owing to their exceptional capabilities [5]. Generally used for monitoring and tracking applications belonging to wide domains ranging from military to farming, habitat monitoring to structural and industrial monitoring. It can also be used for monitoring air pollution, and detecting forest fire and floods. Tracking applications can be tracking of humans, an endangered species or animals in wild life sanctuaries, or any other object of interest. WSN can also be used in health care monitoring to observe condition of disabled and critical patients. Other health care applications are infant health monitoring, blood pressure monitoring \& tracking, firefighter heart rate monitoring and alerting the deaf. WSN has lot of potential to solve real world problems. The future of WSN lies in being aware of the real world and industrial needs, improvising research in those directions and reducing the gap between technology and its real life implementation.

\section{Classification of Wireless Sensor Nodes}

I. Terrestrial WSN: In terrestrial WSN, sensor node can be deployed in preplanned or random way. Sensor nodes are deployed densely and the network is reliable. In preplanned deployment, nodes can be arranged in grid placement, or in $2 \mathrm{~d}$ or $3 \mathrm{~d}$ placement. In terrestrial WSN, data can be communicated effectively in energy efficient way. Various energy minimization techniques, routing protocols, energy harvesting and deployment strategies can be applied for terrestrial WSN [27].

II. Under Ground WSN: In Underground WSN, sensor nodes are placed deep underground or in a cave or mines to monitor underground conditions [30]. Deployment is difficult, expensive and requires careful planning than Terrestrial WSN. Additional sink nodes are required for relaying data to Base station [5]. Battery recharging or Energy Harvesting techniques cannot be implemented in Under Ground WSN [31].

III. Under Water WSN: Under Water WSN consists of sensor nodes and vehicles deployed under water to monitor and track events, such as under water surveillance and exploration, disaster prevention monitoring, under water robotics etc. These sensor nodes are expensive, as they use under water sensors [28]. Under water sensors are susceptible to corrosion, \& have limited bandwidth [5]. Batteries cannot be replaced or re- 
charged. Deployment of sensor node in under water WSN is preplanned and sparse in nature [29].

IV. Multi Media WSN: Multi Media WSN consists of sensor nodes or devices that can store, process and retrieve multimedia data such as audio, images and videos. These types of sensor network require high bandwidth, power and link quality. Sensor nodes are low in cost and have cameras and microphone for capturing audio and video inputs. Deployment of sensor nodes is done in pre planned way to ensure proper coverage [32].

V. Mobile WSN: Mobile WSN is network of sensor nodes capable of moving, self configuring and sensing physical environment [33]. Mobile sensor nodes can provide effective coverage and connectivity than static nodes. Generally used for habitat monitoring, target tracking and military surveillance. Challenges include localization, self configuring and effective coverage of sensing area [5].

\section{Research Issues \& Challenges In WSN}

I. Energy: Sensors are key device for monitoring or tracking systems. Sensor nodes are low in cost, light weight and they have limited battery and power. Energy is an important factor for sensor lifetime. Energy is consumed for node operations such as sensing, data collection and network operations like data communications via different communication protocols. Batteries are small and need to be replaced or recharged, which is not always possible. There are also several methods for producing energy, but they cannot eliminate the need for energy management. The most important challenge is to manage limited battery by designing and implementing various energy efficient hardware and software protocols for WSN. VIII.

II. Harsh Environment Conditions: Sensor nodes are the core element of WSN, are subjected to Radio Frequency (RF ) interface, vibration, highly corrosive environment, high humidity, dirt or dust that degrades their performance [14][15]. Due to these harsh conditions, sensors can malfunction and givqX. inaccurate information to other nodes [16].

III. Self Management: WSN consists of large number of sensor nodes generally deployed statically. But due to failure of nodes WSN topology changes frequently. Also additional sensor nodes can be redeployed at any time which leads to a reorganization of network. Hence, it is required that a sensor network system be adaptable to changing connectivity. During the network operations, the network topology is constantly changing. WSN once deployed should be able to work independently, and must be capable of network configuration, adaption, repair and maintenance [9].

IV. Hardware and Software Issues: Due to tiny size and limited amount of energy source, the nodes have also restricted resources such as CPU performance, memory, communication bandwidth and range. Sensor node can cover a limited area of physical environment; this is termed as its range. The radio range determines the coverage and connectivity for each node. The radio range of node generally lies between 1 to 5 $\mathrm{Km}$.WSN offers various options for CPU implementation microprocessor, microcontroller or Field Programmable Gate Array (FPGA). The performance of sensor node is judged, on the basis of energy consumption per instruction, measured in $\mathrm{nJ} / \mathrm{sec}$. General purpose sensor node has a micro controller. The sensor nodes can generate a huge amount of data, for quick and on time processing. The quantum of processing is different for various kinds of applications. This processing is performed in microcontroller of sensor node. Besides this microcontroller is also responsible for basic WSN functioning, which includes implementing data reception and communication protocols. The computing power of microcontroller should be higher for faster processing [10]. But higher the power more will be the energy consumption. Microcontroller operates in sleep, idle and active states to reduce energy con- sumption. Flash memory is more suitable for sensor network as it is inexpensive, and can persistently store data with no energy to retain data [10]. WSN Software and protocols should be less complex and energy efficient.

V. Heterogeneity: Sensor nodes network can consist of nodes with different sensing, computation and processing capabilities [6]. Heterogeneity arises when two completely different WSN communicate with each other [18]. Heterogeneity can create new issues in communications and network configuration. New routing protocols should be designed and implemented, with the purpose of using sensor node heterogeneity judiciously to increase the lifetime of WSN.

VI. Redundant Data: One of the key objectives of WSN is gathering data from the sensor nodes. Sensor nodes sense data from holistic environment process it and send it to the base station or sink. Sometimes same data is sensed by more than one node, it will cause redundancy of data [22]. Generally sensor nodes are deployed densely; this increases the risk of obtaining duplicate data packets at the sink or base station. It happens as nodes located in closed vicinity sense and transmits same packets [21].This data must not be transmitted to the base or sink node as it will result in wastage of energy or resource [23]. Apart from that, it might happen that the more important data is left behind [24][25].

II. Data Freshness: In WSN, data plays an important role and foremost aim is reliability and security of data. Various WSN applications require real time operations; to achieve this data should reach to sink within the tolerable time limit. Data from previous round should not get mixed with the current round data [19]. It means data freshness should be maintained. But sometimes due to poor network data is not refreshed and incorrect data is transferred to destination.

Event Driven Challenge: The framework of the WSN should be based on Event driven technology [20]. The important data should be given priority as compared to other data. Therefore the framework must be capable detect and report the critical information on time

Quality of Service (QoS): QoS is the measure for competence of Sensor network in meeting application specific requirements [7]. QoS can be defined in two perspectives: application specific and network specific. QoS application specific parameters include network coverage, optimum number of active sensors, quality and accuracy of sensor data measurement, latency and delay. QoS network perspective refers to problem of effectively managing the energy and bandwidth, along with satisfying application requirements. QoS of WSN should support addition and deletion of nodes. Managing QoS parameters for sensor network is difficult as network topology is changing continuously and routing information is imprecise.

X. Deployment: Deployment means implementing sensor nodes in real world scenarios. Deployment can be done in a structured way or randomly, based on the application and demographic location of the area. Sensor nodes can be dropped from helicopters, at hard to reach places. Incase deployed sensor nodes are too dense, network congestion might occur due to many concurrent transmissions attempts made by them. Beside this, all these transmissions might be redundant, as they sense the same phenomenon. Incase deployed sensor nodes are sparse or few, it will result in low data yield or insufficient amount of information [2]. Self configuration property is essential, if nodes are deployed randomly.

XI. Operating System (OS): The OS of sensor must be capable of providing basic memory management and resource management features, but should be less complex as compared to general OS. The OS of sensor node should be capable of working in constraint environment, hardware independent and application specific. It should be energy efficient and capable of taking priority based decisions. Some Operating system designed for sensor node includes Tiny OS, Mantis and Nano-Q plus. 
XII. Security: Security in WSN is one of the key issues in time critical applications, monitoring battlefields, structural monitoring and surveillance applications. In order to protect unauthorized access, the node should support access control. Confidentiality and integrity of data should be maintained [4]. Confidentiality means nodes should encrypt sensed data, prior to its transmission to relay node or base station. For Integrity various mechanism are used so that accurate and original data should reach at base station and finally to the end users. Beside this, due to memory constraints, this security code should be small, so that it can be executed easily by sensors [13]

XIII. Fault Tolerance: The property of fault tolerance implies WSN should remain operational in case of faulty sensors and death of sensor nodes. In WSN, when the power of sensor nodes fall below a certain threshold value, it starts malfunctioning or sometime it is unable to transfer the accurate information to the next node or sink [19]. Sensor networks are prone to power loss; failures and attacks Faulty sensors are the sensors that provide erroneous reading, due to some attack or due to change in environmental condition. These sensors are workable or alive, but provide incorrect measurements. In such critical situations network should remain operational and maintain its minimum serviceability. During WSN operations, sensor nodes deplete energy continuously.

Energy depletion depends on various factors and is uneven for each node. As a result few nodes die early and rest at later part of network lifetime. Beside this, it is infeasible to repair and maintain sensor node after deployment. Sensor network should remain connected and operational, even if few nodes die or few nodes are faulty. Hence, WSN require fault tolerant mechanisms for reliable operation.

XIV. Localization: In many real world scenarios, nodes are deployed randomly, in absence of any supporting infrastructure it is difficult to locate and manage them. The problem of localization deals with learning the physical location of the deployed nodes. Localization is performed with the help location discovery algorithms. These algorithms should be distributed, accurate, robust and scalable in nature [2]. In some cases, they should also support mobility of nodes.

Time Synchronization: Sensor nodes operate independently. Their local clocks are not synchronized with each other. This will result in uncertainty and ambiguity in sensed information. For event detection and time critical applications, real time or time precise operation is mandatory. Some applications give more importance to energy efficiency than accuracy. Hence, based on the type of application, it is important to chose and apply appropriate time synchronization algorithm

\section{Classification of wireless sensor networks}

Table 1: WSN Classification

\begin{tabular}{|l|l|l|l|l|}
\hline Type & No of Nodes & Deployment & Cost & Challenges \\
\hline $\begin{array}{l}\text { Terrestrial WSN } \\
\text { (TWSN)[27] }\end{array}$ & $\begin{array}{l}\text { Hundred to thousand } \\
\text { of inexpensive nodes }\end{array}$ & $\begin{array}{l}\text { Pre planned or } \\
\text { Ad-hoc Manner }\end{array}$ & $\begin{array}{l}\text { In Pre planned TWSN, grid, optimal } \\
\text { and 3D placement models are difficult } \\
\text { to deploy }\end{array}$ \\
\hline Underwater WSN [28][29] & $\begin{array}{l}\text { Several sensor nodes } \\
\text { fewer no than TWSN }\end{array}$ & $\begin{array}{l}\text { Nodes and a Vehicle de- } \\
\text { ployed underwater. Sparse } \\
\text { nodes deployment }\end{array}$ & $\begin{array}{l}\text { More expensive than } \\
\text { TWSN }\end{array}$ & Deployment of nodes \\
\hline Underground WSN[30][31] & $\begin{array}{l}\text { Less no of nodes than } \\
\text { TWSN }\end{array}$ & $\begin{array}{l}\text { Meployed in cave or mines } \\
\text { TWSN in terms of than } \\
\text { equipments and mainte- } \\
\text { nance }\end{array}$ & $\begin{array}{l}\text { Deployment is difficult, collection of } \\
\text { information }\end{array}$ \\
\hline Multimedia WSN[32] & $\begin{array}{l}\text { Several sensor lower } \\
\text { cost nodes having } \\
\text { cameras and micro- } \\
\text { phones }\end{array}$ & $\begin{array}{l}\text { Deployed in pre planned } \\
\text { aay to ensure proper cover- } \\
\text { age } \\
\text { Effective deployment makes } \\
\text { data retrieval and compres- } \\
\text { sion easy }\end{array}$ & Very costly & $\begin{array}{l}\text { High bandwidth demand, high energy } \\
\text { consumptions QOS provisioning, } \\
\text { cross layer design, compression tech- } \\
\text { niques }\end{array}$ \\
\hline Mobile WSN [33] & $\begin{array}{l}\text { Deployed in large } \\
\text { numbers }\end{array}$ & $\begin{array}{l}\text { capable of moving, self } \\
\text { configuring and sensing } \\
\text { physical environment }\end{array}$ & Very costly & $\begin{array}{l}\text { Localization, Self configuring, De- } \\
\text { ployment and effective coverage of } \\
\text { sensing area }\end{array}$ \\
\hline
\end{tabular}

\section{Conclusion}

WSN is at its developing phase, and there are various issues that still need to be identified. WSN is applied to varied applications, each having its specific requirement; hence a thorough investigation is required. In this paper, only some of the issues that have been experienced by the researcher are discussed. For practical implementation of WSN, energy saving is major concern in the resource constraint environment. In terms of hardware, sensor network must be scalable, and capable of fulfilling QoS requirement, on software front the algorithms and protocols used should be energy efficient. WSN has emerged as an active research area, involving various challenging topics such energy consumption, routing algorithms, deployment and localization problems.WSN has vast potential; the need of the hour is to utilize and manage it effectively.

\section{References}

[1] K. Akkaya \& M. Younis, "A survey of Routing Protocols in Wireless Sensor Networks", Adhoc network Journal, Elsevier, 2005, pp 325-349.
[2] S.Gowrishankar, T.G.Basavaraju, Manjaiah D.H, and Subir Kumar Sarkar, "Issues in Wireless Sensor Networks", Proceedings of the World Congress on Engineering, vol. I, July, 2008, London, U.K.

[3] Sukhwinder Sharma, Rakesh Kumar Bansal and Savina Bansal, "Issues and Challenges in Wireless Sensor Networks", International Conference on Machine Intelligence Research and Advancement IEEE, 2013

[4] Yide Liu, "Wireless Sensor Network Applications in Smart Grid: Recent Trends and Challenges", International journal of Distributed Sensor networks, 2012

[5] Jennifer Yick, Biswanath Mukherjee and Dipak Ghosal, "Wireless sensor network survey", Computer Networks, Vol 52, Elsevier, 2008

[6] J.Sen, "A survey on wireless sensor network security," International Journal of Communication Networks and Information Security, Vol 1 no.2 pp 59-82, 2009

[7] R.Mulligan and H.M.Ammari, "Coverage in Wireless Sensor Networks: A Survey", Network Protocols \& Algorithms, vol.2, no. 2, 2010, pp 27-53.

[8] M.K.Jain, "Wireless Sensor Networks: Security Issues and Challenges", Internal Journal of Computer and Information technology vol 2 no.1, 2011, pp62-67

[9] A.Jangra, Swati, Richa and Priyanka, "Wireless Sensor etwork (WSN): Architectural Design issues and Challenges", International Journal on Computer Science and Engineering, vol 2, no 9, 2010 , pp 3089-3094

[10] "Applications of Wireless Sensor Networks in Next Generation Networks”, ITU-T Technical paper, NGN-AWSN (2014-02). 